\title{
Update on Minimal Standards for Electroencephalography in Canada: A Review by the Canadian Society of Clinical Neurophysiologists
}

\author{
Dianne Dash, Chelsea Dash, Sara Primrose, Lizbeth Hernandez-Ronquillo, \\ Farzad Moien-Afshari, Lady D. Ladino, Juan Pablo Appendino, Lindsay Mazepa, \\ Christine Elliott, Seyed M. Mirsattari, Paolo Federico, Esther Bui, Gary Hunter, \\ Rajesh RamachandranNair, Rohit Sharma, Paula Melendres, Joanne Nikkel, \\ Dang Khoa Nguyen, Salah Almubarak, Mike Rigby, Jose F. Téllez-Zenteno
}

\begin{abstract}
Surface electroencephalogram (EEG) recording remains the gold standard for noninvasive assessment of electrical brain activity. It is the most efficient way to diagnose and classify epilepsy syndromes as well as define the localization of the epileptogenic zone. The EEG is useful for management decisions and for establishing prognosis in some types of epilepsy. Electroencephalography is an evolving field in which new methods are being introduced. The Canadian Society of Clinical Neurophysiologists convened an expert panel to develop new national minimal guidelines. A comprehensive evidence review was conducted. This document is organized into 10 sections, including indications, recommendations for trained personnel, EEG yield, paediatric and neonatal EEGs, laboratory minimal standards, requisitions, reports, storage, safety measures, and quality assurance.

RÉSUMÉ: Mise à jour des normes minimales relatives à l'encéphalographie au Canada. L'utilisation d'un électroencéphalogramme (EEG) reste la méthode de référence pour évaluer de façon non invasive l'activité électrique cérébrale. Il s'agit du moyen le plus efficace pour diagnostiquer et catégoriser les syndromes épileptiques mais aussi pour définir l'emplacement des foyers épileptogènes. L'EEG est ainsi utile lorsqu'il s'agit de décider d'un traitement à donner et d'établir un pronostic dans le cas de certains types d'épilepsie. L'électroencéphalographie est aussi un domaine en évolution dans lequel de nouvelles méthodes sont introduites. La Société canadienne de neurophysiologie clinique a du coup constitué un comité d'experts afin d'élaborer, à l'échelle nationale, de nouvelles normes minimales. Pour ce faire, un examen exhaustif fondé sur des données probantes a été mené. Le document qui en a résulté est divisé en 10 sections : directives et recommandations destinées à du personnel qualifié, efficacité des EEG, EEG pédiatriques et néonatales, normes minimales en laboratoire, demandes d'EEG, production de rapports, conservation, mesures de sécurité et assurance de la qualité.
\end{abstract}

Key Words: Electroencephalogram, guidelines, national, protocol, quality assurance, standards, EEG-computer applications doi:10.1017/cjn.2017.217

Can J Neurol Sci. 2017; 44: 631-642

\section{BACKGROUND}

Electroencephalography (EEGy) is one of the earliest ancillary tests developed in neurology and the neurosciences. The first description of this procedure in humans was published in 1929. However, technological changes in recent decades have led to vast improvements in the recording and reviewing quality of the electroencephalogram (EEG), in addition to storage demands and capabilities and data transmission over long distances. ${ }^{1}$ Currently, EEGy remains the gold standard for analyzing electrophysiological processes involved not only in epilepsy but also in other central nervous system (CNS) disorders. ${ }^{2}$

The EEG is a unique tool because of its ability to detect the collective electrical activity generated by cortical neurons with unequalled temporal resolution. ${ }^{3,4}$ Furthermore, it is a noninvasive, relatively affordable, and easy-to-perform test that can be repeated several times, allowing close patient follow-up, and it provides a dynamic vision of CNS disorders. ${ }^{5}$ It is necessary for the classification of epilepsy syndromes as well as in determination of the epileptogenic zone, especially in cases where surgery is being considered. ${ }^{6}$

From the Royal University Hospital, Saskatoon, Saskatchewan, Canada (DD, CD, SP); Department of Medicine, Division of Neurology, University of Saskatchewan, Saskatoon, Saskatchewan, Canada (LHR, LDL, GH, JFTZ); University of British Columbia, Vancouver, British Columbia, Canada (FMA); University of Calgary, Calgary, Alberta, Canada (JPA, PF); British Columbia Institute of Technology, Vancouver General Hospital, Vancouver, British Columbia, Canada (LM); Queen Elizabeth II Health Sciences Center, Halifax, Nova Scotia, Canada (CE); Western University, London, Ontario, Canada (SMM); University of Toronto, Ontario, Canada (EB); McMaster University, Hamilton, Ontario, Canada (RRN); Sick Kids Hospital, Toronto, Ontario, Canada (RS); Health Sciences Center, Winnipeg, Manitoba, Canada (PM, JN); University of Montreal, Montreal, Quebec, Canada (DKN); Department of Paediatrics, Saskatoon Health Region, Saskatoon, Saskatchewan, Canada (SA); Foothills Medical Centre, Calgary, Alberta, Canada (MR).

Received January 6, 2017. Final Revisions Submitted April 12, 2017. Date of ACCEPTANCE APRIL 18, 2017.

Correspondence to: José F. Téllez-Zenteno, MD, PhD, Department of Medicine, Division of Neurology, Royal University Hospital, Saskatoon, Saskatchewan S7N 0W8, Canada. E-mail: jose.tellez@usask.ca OR jftellez@yahoo.com 
Fourteen years have passed since the Task Force of the Canadian Society of Clinical Neurophysiologists (CSCN) published the Canadian electroencephalography standards, an initial set of guidelines for EEGy in Canada. ${ }^{7}$ We now present an updated version of the minimal standards for the practice of conventional EEGy. These guidelines will not specify equipment or protocols that must be used; rather, we discuss the available technologies in terms of diagnostic and clinical value and make recommendations concerning the indications for which EEGy is best suited. The purpose of these minimal standards will serve as a benchmark for further development, standardization, and quality care in clinical neurophysiology laboratories in Canada.

\section{Methods}

\section{Review of the Literature}

The EEG Guidelines Project began at the end of 2013. A comprehensive literature search was done on the Medline ${ }^{\circledR}$, Embase $^{\circledR}$, Index Medicus ${ }^{\circledR}$, and the Cochrane databases from January of 1980 to July of 2015. The search incorporated Medical Subject Headings and text words for literature on Standards for Electroencephalography. Key search words included, but were not limited to, continuous EEG (cEEG), EEG, electrodes, electroencephalography, electroencephalogram, epilepsy monitoring unit, guideline, indications, montage, parameters, policies, protocol, recommendations, recording, reporting, routine EEG, safety, sleep-deprived EEG, short-term video EEG, standards, storage, task force, technical, telemetry, video EEG, quality assurance, policy, and procedures.

We also reviewed the bibliographies of reviews, original articles, and book chapters, as well as documents related to the "Standards for Electroencephalography" previously published by the American Academy of Neurology (AAN), the American Clinical Neurophysiology Society (ACNS), the American Electroencephalographic Society (AEEGS), the American Epilepsy Society (AES), the National Association of Epilepsy Centers (NAEC), the Canadian Association of Electroneurophysiology Technologists (CAET), the American Society of Electroneurodiagnostic Technologists (ASET), the National Institute for Health and Clinical Excellence (NICE), and the International League Against Epilepsy (ILAE).

We included studies published in English if they contained original research involving $\geq 30$ patients. Documents were included if the main subject pertained to indications, parameters, activation techniques, guidelines, or protocols about the practice of EEGy. We included patients irrespective of age. Children were considered to be those under 16 years old. We excluded studies with non-consistent data or any overlapping patient populations from the same centre. A total of 1,655 articles were initially identified; 196 were included for full-text screening; and 61 articles, websites, and other documents were included.

\section{Panel of Experts}

The CSCN convened a national expert panel to develop new and updated national minimal guidelines. It included a panel of clinical neurophysiologists, electroencephalographers, epileptologists, and EEG technologists from across the country. The expert panel reviewed the recommendations in the 2002 guidelines $^{7}$ and suggested additional topics to be researched. At this meeting, work teams were formed to discuss and develop the topics of this document. In 2015, three teleconferences with the CSCN executive and membership were carried out, and all of the statements were integrated into a single document. The entire document was prepared in 2016 in accordance with the CAET minimal technical standards and organized into 10 sections. The new guidelines added literature related to the era of digital media, including such new practical sessions as indications: EEG yield and activation procedures, cEEG, video, neonatal and paediatric EEG, digital EEG media, electrodes, montages, and filters. The new guidelines additionally update the previous sections: electroencephalographers, technologists, laboratory standards and procedures, EEG requisitions, records and reports, informed consent, and quality improvement. ${ }^{7}$ The experts tried to simplify the guidelines as much as possible.

\section{Results}

\section{General Indications}

The main indication for performing an EEG is the clinical suspicion of epilepsy. The possibility of a seizure is considered for a patient who reports paroxysmal clinical events involving sensory, motor, and psychic phenomena, including alteration of consciousness. However, because of the unpredictable and intermittent nature of seizures, there is only a small chance of recording the patient's stereotypic events during a routine EEG recording. ${ }^{8}$ Interictal epileptiform discharges (IEDs) are highly correlated with the diagnosis of epilepsy, and their presence on an EEG performed after a single seizure has been found to be helpful in predicting the risk of seizure recurrence. ${ }^{9}$ However, it is important to bear in mind the prevalence of IEDs in healthy populations. IED prevalence is about $1 \%$ in healthy infants (children younger than 5 years) and $6.5 \%$ in healthy children (6-13 years). Similarly, the rates of spontaneous IEDs in healthy adult volunteers vary from 0.1 to $6.6 \%$ (So, 2010). ${ }^{10,11}$

EEGy not only provides support for the diagnosis of epilepsy but is also instrumental in the classification of seizures and specific epilepsy syndromes, as well as disease follow-up. ${ }^{12}$ Likewise, the EEG plays a primary role in the diagnosis and management of status epilepticus. ${ }^{13}$ EEGy is also useful in the detection of the most common differential diagnoses of epileptic seizures: psychogenic non-epileptic seizures (PNESs). EEGy may be the only modality that can distinguish an epileptic seizure from a PNES. ${ }^{12}$ Furthermore, its utility has been demonstrated in the assessment of comatose patients or with regard to changes in level of consciousness. For example, generalized periodic discharges (GPDs) with triphasic morphology waves may point to hepatic encephalopathy, and an excess of beta activity may suggest a barbiturate or benzodiazepine overdose. ${ }^{13,14}$

Focal slowing visible on EEG recordings can sometimes reveal altered physiology in a specific brain region that would not be detected on structural imaging. ${ }^{12}$ For instance, in cases of subcortical (diencephalic) alterations, intermittent rhythmic delta activity can occur. EEGy is also useful in establishing neurological prognosis after cardiorespiratory arrest ${ }^{3,15}$ and is one of the modalities available for assessing brain death, although now rarely used. $^{16}$

There is some evidence that electrophysiological profiles may be associated with different neurological disorders. ${ }^{17}$ Certain EEG patterns may point toward specific underlying causes such as Creutzfeldt-Jakob disease, anti-N-methyl-D-aspartate 
(anti-NMDA) encephalitis, ${ }^{15}$ leucine-rich glioma inactived-1 (LG11) antibody-mediated encephalitis, ${ }^{14}$ herpes simplex encephalitis (HSE), ${ }^{18}$ or focal cortical dysplasia type II. ${ }^{19}$ Slowing of EEG rhythms that accompany delirium and dementia can also progress as disease advances, providing a modality for diagnosing and monitoring disease severity. ${ }^{5,12,20}$ For psychiatrists, EEGy can be useful in clinical scenarios where there is a suspected interplay between functional symptoms and organic aetiology. Psychiatrists should base their use of EEGy on robust clinical assessment and should be aware of the limitations inherent in the procedure. $^{21}$

\section{Trained Personnel}

The healthcare workforce should be sufficiently prepared to provide accurate diagnostic services and patient-centred care that meets the patient's (and family's) needs. ${ }^{7}$

\section{Standards for Electroencephalographers}

The national standard for electroencephalographers in Canada is the qualifying examination developed by the EEG section of the CSCN. The CSCN strongly recommends that provinces that do not have a provincial qualifying examination use the successful passing of this examination as a prerequisite for the practice of interpreting EEGs. All electroencephalographers entering EEGy practice should have the following qualifications: ${ }^{6}$

1. The individual should be a licensed neurologist or neurophysiologist within the province of practice and should be recognized as a specialist in neurology by the Royal College of Physicians and Surgeons of Canada or the Provincial Medical Licensing College.

2. The electroencephalographer should have completed a minimum of six months of full-time supervised training in a laboratory that is directed by a full member of the CSCN or of an international equivalent. ${ }^{7}$

It is recommended that continuing educational activities be in keeping with maintenance of the Certification Program of the Royal College of Physicians and Surgeons of Canada. Individual regular review of journals and attendance at related conferences are strongly encouraged. It is also recommended that the electroencephalographer regularly meet with technologists for reading sessions and, if possible, other electroencephalographers in the area. 6,7

\section{Laboratory Director}

Each EEG laboratory should appoint a medical director, a physician who meets the CSCN qualifications for the practice of EEGy. The responsibilities of the director should include the following: ${ }^{7}$

1. Provide continuous, adequate, and effective direction and supervision of the manager and supervising EEG technologist, who is responsible for the daily operation of the laboratory and for the laboratory staff. ${ }^{6}$

2. Maintain the highest standards of EEG technical practice, together with the manager and supervising EEG technologist.

3. Ensure that a quality-assurance or quality-improvement program is in place. ${ }^{7,22}$

4. Document the policies of the laboratory in a policy and procedures manual.

5. Provide the documentation necessary for accreditation upon request.

\section{EEG Technologists}

\section{Accreditation}

The Canadian Board of Registration of Electroencephalographic Technologists Inc. (CBRET) is the national organization that provides a qualifying registration examination. ${ }^{7,23}$ The CSCN recommends passing the CBRET examination as the national standard for qualification as an EEG technologist. It is recognized that provincial/regional authorities have the final authority in setting standards for EEG technologists for that province/health region. ${ }^{7}$

\section{Continuing Medical Education}

It is recommended that EEG technologists maintain and improve their knowledge and skills by reading journal articles and textbooks related to the field of EEGy, attend didactic courses in clinical neurophysiology, and participate in EEG "rounds" and department conferences. ${ }^{6}$ It is suggested that medical directors of clinical EEG laboratories promote continuing education for their laboratory staff and assist them in successful completion of board examinations. ${ }^{6}$ In addition, regular reading sessions and regular attendance at provincial, Canadian, and international meetings are encouraged. ${ }^{6}$ Funding for such continuing education should reside with the health facility. ${ }^{6}$ It is recommended that technologists be certified in cardiopulmonary resuscitation and recertified every two years. Finally, it is recommended that they have membership in the CAET as well as the provincial technologist organization if applicable. ${ }^{7,22}$

\section{Professionalism}

Professional conduct should be in keeping with the Code of Ethics of the CAET and the Core Entry-to-Practice Competencies for the Profession of Electroencephalography Technology. ${ }^{22,24} \mathrm{At}$ all times, confidentiality of patient records should be maintained. $^{22,24}$

\section{Long-Term Monitoring for Epilepsy (LTME)}

Personnel involved with LTME are encouraged to have specialized training due to its technically more challenging nature. Such training should involve: the use and maintenance of equipment, safety protocols, management of seizures and seizurerelated emergencies, recognition of ictal and interictal electrographic patterns, artefactual signals, data retrieval, reduction, and storage. $^{25}$ Additionally, technologists involved with intracranial recordings should have specialized training in interpreting intracranial EEG, operating room procedures, and sterile technique.

It is recommended that the laboratory director have specialized training (preferably an epilepsy or neurophysiology fellowship training in a centre with experience doing LTME) in the operation of LTME equipment and infrastructure (recording, storage, and retrieval) and in interpretation of data. The LTME electroencephalographer should have similar training or be under the direction of the laboratory director.

\section{ICU EEG Monitoring}

Specialized training is recommended for personnel involved with cEEG monitoring of critical-care patients, which are accompanied by distinct technical and interpretation challenges. 
The qualified electroencephalographer and EEG technologists involved in intensive care unit (ICU) monitoring should be knowledgeable with respect to the procedures and equipment, medical terminology, and electrophysiological patterns/abnormalities/artefacts more specific to, or more commonly found in, ICU EEG monitoring. ${ }^{7,26,27}$ As per CAET standards, the technologist should annotate current medications, including anaesthetics, body temperature, and the use of hypothermia. The technologist must perform stimulation procedures (i.e., visual, auditory, somatosensory, painful, passive eye opening/closing) to assess EEG reactivity. ${ }^{22}$ While most seizures in the ICU have minimal clinical features, continuous-video EEG should be performed to assist with identifying artefacts and subtle clinical changes. Continuous recordings should employ such quantitative analysis tools as a compressed spectral array whenever possible in order to demonstrate trends over time and graphically represent long recordings in easy-to-understand formats, which can facilitate communication with intensivists and nurses, as well as reduce reviewing time. Electrodes should be checked at a maximum interval of every 24 hours, or more frequently based on individual patient factors and the quality of recordings.

\section{Ambulatory EEG}

Specialized training is encouraged for the technologist and electroencephalographer due to its technically more challenging nature. Furthermore, utilization of ambulatory EEG (AEEG) recording may be considered as an alternative to inpatient cEEG/ LTME. This procedure has been shown to be effective in differentiating between epileptic and non-epileptic events, especially in children whose spells occur on a daily basis. ${ }^{28}$ Seizure semiology should be carefully considered when referring a patient for an AEEG, as certain seizure types may show minimal or no change on scalp EEGs. AEEG is most beneficial in capturing IEDs, subclinical seizures, sleep/wake differentiation, or activity that does not require simultaneous video recording for diagnosis. A study performed in adult patient $\mathrm{s}^{29}$ showed a diagnostic yield of $72 \%$ with the following indications: characterization of possible PNES, clarifying the diagnosis of epilepsy that is not clear, and quantification of spikes and seizures. In paediatric patients, the yield of AEEG to differentiate seizures from PNES events, to determine seizure/interictal discharge frequency, and to classify seizure type or localization has been reported from 61 to $100 .^{28}$

\section{EEG YIELD}

An initial routine EEG recording in a patient with epilepsy will have no epileptiform activity in about $50 \%$ of cases. ${ }^{30}$ EEGy has relatively low and variable sensitivity (25-56\%) and moderate specificity (78-98\%). ${ }^{6}$ Sensitivity increases with repeat or prolonged recordings and may be higher for some seizure types (e.g., absence or juvenile myoclonic epilepsy) than others (e.g., temporal lobe seizures). When a routine EEG fails to show IEDs in a patient with a suspected seizure disorder, repeating the EEG and using additional activation procedures such as sleep deprivation (SD) and repeating hyperventilation (HV) or intermittent photic stimulation (IPS) may increase diagnostic sensitivity. ${ }^{8}$ It is important to recognize that a normal EEG does not exclude epilepsy, as approximately $10 \%$ of patients with epilepsy never show IEDs on routine EEGs. Additionally, an abnormal EEG demonstrating IEDs does not itself indicate that an individual has epilepsy. ${ }^{6}$

\section{Activation Procedures}

Activation procedures such as HV and IPS should be routine practice. ${ }^{7}$ It is good practice to warn patients of the small risk of seizure induction and the temporary side effects, and to obtain consent prior to these procedures. ${ }^{6}$ Minimally, the baseline EEG should consist of 20 minutes of artefact-free recording in addition to the time needed to perform activation procedures. Occasionally, longer recordings may be necessary to adequately assess the presence of IEDs. ${ }^{22}$ If activation procedures are discontinued prematurely or omitted, the reasons should be documented on the recording and in the technical report.

\section{Hyperventilation}

An HV procedure can activate IEDs and, sometimes, ictal EEG discharges ${ }^{8}$ in patients with absence seizures as well as in focal epilepsies. ${ }^{9}$ An HV protocol should be executed unless contraindicated. HV is contraindicated in patients with the following medical conditions: recent brain surgery, stroke, intracranial haemorrhage, uncontrolled hypertension, severe cardiovascular or pulmonary disease, exertion-induced asthma, brain tumour, moyamoya disease, sickle-cell disease, or trait or another cause of blood hyperviscosity. It should also be avoided in patients with an inability or unwillingness to cooperate (intellectual disability, dementia). The contraindication is selective during pregnancy, and clinical judgment should be used for patients over the age of 65. Obtaining an adequate history, which may contraindicate HV, is often difficult with patients from nursing homes and assistedliving facilities. Precaution with HV should be higher in the elderly. ${ }^{6}$ Finally, HV can be excluded if there is already abundant epileptiform activity on the pre-HV portion of the recording,

If no significant EEG changes, outside of normal buildup, are noted during the routine three minutes of $\mathrm{HV}$, and there is a strong suspicion of absence epilepsy, the protocol should be prolonged and/or repeated according to laboratory standards. ${ }^{9,22,31}$ If there is unexpected abnormal hypersynchronization activity during $\mathrm{HV}$, the possibility of hypoglycaemia or other metabolic abnormalities or simply that the patient may not have stopped hyperventilating should be excluded. ${ }^{30}$ The technologist should always write down an assessment of the quality of patient effort periodically throughout this procedure as well as in the technologist report. ${ }^{22,32,33}$ The elapsed time should be clearly indicated on the recording at 30 -second intervals. ${ }^{22}$

\section{Intermittent Photic Stimulation}

IPS should be performed in accordance with current CAET standards. ${ }^{22,34}$ The IPS protocol should be executed or omitted if contraindicated according to the IPS CAET minimal technical standards. Events or responses should be documented on the EEG at the time of their occurrence. Attempts to confirm sensitivity to a particular frequency should be separated in time to prevent specific habituation of the responses. Electromyogram (EMG) electrodes may be necessary to detect myoclonus during a photoparoxysmal response (PPR). Photic stimulation should be stopped immediately if any generalized epileptiform activity occurs. Additional attempts at that specific frequency may be 
required to confirm photosensitivity. ${ }^{35}$ Because of the risk of provoking a seizure, IPS should be avoided in pregnancy. ${ }^{35}$

\section{Sleep Deprivation}

When a standard EEG fails to show epileptiform activity and a seizure disorder is suspected, an SD-EEG should be obtained. ${ }^{30}$ At a minimum, sleep should be half the time of the normal sleep pattern, unless otherwise indicated by the referring physician or laboratory protocol. It is known that sleeping during an inappropriate circadian phase (i.e., in the morning) enhances sleep instability and causes the occurrence of epileptiform discharges. ${ }^{36,37}$ At least 30 minutes of artefact-free recording should be obtained. Additional time is required for a period of wakefulness before and after sleep, plus HV and IPS, unless contraindicated. When undergoing an SD-EEG, patients should be informed that they are not allowed to drive to and from their EEG appointment and should therefore always be accompanied.

\section{Video}

Simultaneous video recording with EEGy is a useful addition, and it is strongly recommended, though it is not mandatory in a routine 30-minute EEG. In some circumstances, video is required. When suspecting abnormal movements, it is necessary to perform an EMG of relevant muscles coupled with video recording. In cEEG at an ICU and in LTME, video recording is essential, with strict synchronization of the EEG signal and video. The video is useful for interpreting clinical events as well as identifying artefacts; however, the use of video does not reduce the importance of having an attentive technologist. ${ }^{38}$

\section{Continuous EEG}

cEEG typically refers to video EEG recordings in order to monitor for seizure activity and in general continues for 24 hours or more. The yield of capturing epileptiform (seizures or IEDs) is dependent upon aetiology, as well as the patient's clinical state and age. As a general rule, the chances of capturing seizures or IEDs increases with duration of cEEG recording. In critically ill children, a 24-hour recording will capture $72-80 \%$ of seizures, while a 1-hour recording will do so in $50 \%$ of cases. ${ }^{39,40}$ In one study of adults, ${ }^{41}$ combined with a small proportion of children, a higher yield of detecting seizures in the first 24 hours of recording was seen in non-comatose (95\%) compared to comatose patients $(80 \%)$. In critically ill patients, cEEG should be recorded for at least 24 hours following cessation of seizures when medication changes are no longer needed (titration or weaning).

The ACNS has issued recommendations for the use of cEEG, ${ }^{14}$ which are as follows: (1) to identify electrographic seizures and status epilepticus in those patients who have been treated without improving their level of awareness, in the context of preexisting epilepsy, traumatic brain injury, intracranial haemorrhage or ischemic stroke, post-cardiac arrest, central nervous system infection, or metabolic encephalopathy (including congenital errors of metabolism); (2) to characterize paroxysmal events of uncertain aetiology; and (3) to assess the efficacy, titration, and weaning of the therapy in frequent seizures or status epilepticus. cEEG has also been suggested in the use of prognostication; however, its benefits compared to periodic regular EEGs have not yet been established.

\section{Neonatal and Paediatric Eegs}

It is highly recommended that all neonatal (up to 8 weeks postterm) and paediatric (8 weeks post-term or greater) EEGy recordings follow the CAET standards for additional activation processes in order to evaluate EEG reactivity. This should include tapping and auditory, visual, painful, and somatosensory stimuli. $^{22,30}$

\section{Neonatal}

EEGy helps to determine the level of maturation of the brain in neonates. ${ }^{30}$ With the increasing number of premature infants, particularly the extremely preterm newborns less than 27 weeks gestational age at birth, EEGy has become a useful tool for diagnosis and assessment of prognosis of brain injuries. ${ }^{42}$ Neonatal seizures should be diagnosed with the help of ictal EEG findings. EEGy is also useful in elucidating the timing and mode of brain injuries in the preterm newborn. Regardless of aetiology, the efficacy of treatment should be evaluated using cEEG monitoring in the acute stages of brain insult. ${ }^{43}$

\section{Paediatric}

In patients where IEDs occur mostly during sleep or at sleep-wake transitions, it is essential that a portion of the EEG recording be obtained during these states. It is essential that $\mathrm{HV}$ be performed during the EEG in children with suspected absence seizures in the absence of contraindications. ${ }^{44}$ The technologist should make careful annotations about the patient's responsiveness.

\section{Laboratory Standards ANd Procedures}

\section{EEG Equipment and Digital Media}

It is recommended to employ a minimum of 16 channels so as to improve localization and thereby facilitate accurate interpretation. However, if possible, the use of a larger number of channels (24-32) is encouraged. Additional derivations may include: electrooculogram (EOG), electrocardiogram (ECG), electromyography (EMG), pulse oximetry of submental muscles, oral/nasal thermistors, or a strain gauge for respiratory monitoring. A single-channel ECG should be included on one EEG channel. This is helpful in distinguishing between epileptiform discharges and ECG artefacts, so as to identify pulse and movement artefacts. $^{45,46}$ All ancillary equipment must have initial approval of the Canadian Standards Association (CSA) and undergo subsequent annual maintenance checks. ${ }^{22}$ All equipment must be CSA-approved. Yearly checks and documentation on equipment safety must be maintained.

\section{Digital EEG Media}

A digital EEG recording system offers multiple advantages over an analog/paper system: higher-fidelity recording; signal post-processing with control over the montage, filter settings, gain, and horizontal scaling; and efficient data storage are some of the main advantages. Acquisition of EEG data into a digital storage medium should occur at a minimum sampling rate of 256 per second. Digitization should use a resolution of at least 16 bits per sample, including any sign bit. The common mode rejection ratio should be at least $90 \mathrm{~dB}$ for each of the channels. Additionally, video recording should be synchronized with the 
EEG to facilitate review, especially for identification of artefacts and clinical events. ${ }^{47}$

Standard horizontal scaling should be available in which 1 second occupies between 25 and $35 \mathrm{~mm}$, with a minimum resolution of 128 data points per second on the screen for a 10 -second page, requiring a horizontal resolution of at least 1,280 pixels. A standard vertical scaling with a minimum spacing of $10 \mathrm{~mm}$ per channel should be employed for a display of up to 21 channels. $^{47}$ The system should allow for simultaneous display of multiple segments of the EEG, allowing side-by-side visual comparison of different segments within one recording as well as different segments from different recordings obtained on different days. $^{48}$

\section{Electrodes}

Surface disk electrodes are recommended for routine clinical use. All electrodes, whether used for routine or otherwise, must adhere to CAET technical standards. ${ }^{22}$ Inter-electrode impedances must comply with CAET standards. Ordinarily, all electrode impedances should be equal and not exceed $5000 \Omega$ (ohms) in routine settings. In LTME and cEEG, where electrode application is prolonged and patient skin is susceptible to breakdown, it is more important to have balanced electrode impedances that do not exceed the upper limit of 10,000 $\Omega$. Electrode impedance should be rechecked during the recording when any pattern that might be artefactual appears. ${ }^{32}$

Electrode application using the 10-20 system should be employed. ${ }^{22}$ For routine EEGs, the 10-20 system may be adequate for most patients and efficient in terms of time, effort, and cost. On the other hand, the additional, more closely spaced electrodes in the 10-10 system clearly provide better spatial resolution. In patients undergoing pre-surgical evaluation, they can provide precise localizing information with regard to IED and ictal EEG onsets. However, placement of several additional electrodes requires increased time and effort on the part of technologists, potentially reducing the number of studies that can be performed in a day. Finally, additional electrodes need to be purchased, thus increasing the cost. ${ }^{45}$

\section{Recording}

All recordings must meet current CAET minimal technical standards. Appropriate calibrations should be made at the beginning of every EEG recording. This includes at least 10 seconds (or the duration needed to reach a stable recording) of a square wave calibration. ${ }^{38}$ Some EEGs may need to be reviewed and reported by an electroencephalographer more quickly than within the normal timeframe. Using their best judgment and erring on the side of patient safety, technologists should notify the appropriate electroencephalographer about significant findings that require urgent attention.

\section{Montages}

Montage reformatting in record review mode is recommended to enhance signal appreciation; however, the recording should be performed using montages that enhance abnormalities to facilitate clear, obvious, and fast interpretation by the electroencephalographer. Montages should be designed for adequate spatial sampling, utilizing the International 10-20 System of Electrode Placement. The 10-20 system is the only one officially recommended by the International Federation of Clinical
Neurophysiology. The ACNS published a proposal for montages to be employed in EEGy for standard use by clinical laboratories. 49

Routine clinical EEG recordings should include a minimum of longitudinal bipolar, transverse bipolar, and referential montages, and the recording should always begin on a longitudinal bipolar montage. A logical order of arrangement should prevail in each montage. The electrode connections for each channel should be clearly indicated at the beginning of each montage, the electrode connections should be made as simple as possible, and montages should be easily comprehended. Tracings from the more anterior electrodes should be placed above those from the more posterior electrodes on the recording page; similarly, a "left-above-right" order of derivations should be utilized. Mild modifications of the recommended montages may be applied for monitoring other physiologic variables, so long as these modifications do not conflict with the principles set forth in the previous recommendations. ${ }^{45,46}$

The limitations of the 10-20 system for precise localization of temporal activity have been recognized for years, leading to the use of additional noninvasive (T1/T2 electrode positions) and semi-invasive electrodes (nasopharyngeal, sphenoidal, and foramen ovale electrodes). However, the use of the temporal electrode positions described in the 10-10 system (FT7/FT8, FT9/FT10, T9/T10) can be helpful and may obviate the need for T1/T2 and invasive electrodes. The positions of the FT9/FT10 electrodes closely approximate those of the T1/T2 electrodes. Anterior temporal electrodes detect interictal and ictal epileptiform abnormalities virtually as well as do sphenoidal electrodes. They also provide more consistent recording information, do not result in pain and discomfort for patients, and do not require physician expertise. $^{46,50}$ We therefore recommend always applying electrodes inferior to the temporal standard 10-20 system of electrode placement.

\section{Filters}

For digital recordings, filtering of the signal occurs at two levels. Analog filters are applied to the incoming signal in the actual amplifier before digitization. These are dependent on the specific amplifier being used and are not modifiable by the user. The second level of filtering comprises digital filters that are applied before display of the digitized data. The use of these filters does not permanently alter the recorded data; it only processes the data for display. ${ }^{38}$ For standard recordings, the low-frequency filter should be no higher than $1 \mathrm{~Hz}$. A low-frequency filter setting higher than $1 \mathrm{~Hz}$ could attenuate slow-wave activity in the record. Vital information may be lost when pathologic activity in the delta range is present. The high-frequency filter should be no lower than $70 \mathrm{~Hz}$, as a setting lower than $70 \mathrm{~Hz}$ for high-frequency filters can distort, attenuate, or transform spikes and other pathologic sharp features into unrecognizable forms and can cause muscle artefacts to resemble spikes. ${ }^{38}$ Making selective filter setting changes in review mode is encouraged to improve signal detection and enhance signal appreciation. ${ }^{32}$ The $60-\mathrm{Hz}$ (notch) filter should not be used in routine clinical settings. Its use should be restricted to hostile recording environments (i.e., the ICU), where $60-\mathrm{Hz}$ interference cannot be readily eliminated by proper troubleshooting techniques. ${ }^{22}$ The use of a notch filter may mask a serious safety hazard and risk the loss of low-amplitude spike potentials. $^{30,32}$ 


\section{Patient Information}

As a minimum, the digital file should include the patient's name, date of birth, identification number, and test date. In addition, medications, relevant clinical history and observations, hand dominance, time of last nourishment, skull anomalies, name of the technologist, and the technologist's impression should be noted. ${ }^{7,22,30,32}$

\section{Recording Annotations}

The EEG technologist should use standard medical terminology when documenting relevant information on the recording and when preparing a written technical impression for the interpreting physician. Abbreviations used for annotations should be standardized within each laboratory. Technical, clinical, and behavioural changes should be indicated on the recording at the time of their occurrence. Signals or commands to the patient, the presence or absence of clinical responses to stimuli, onset and conclusion of activation procedures, and movements should also be annotated. Careful observation of the patient with frequent notations is essential, particularly when unusual waveforms are observed in the tracing. ${ }^{32}$

\section{Technical Impression}

The EEG technologist should prepare a comprehensive technical impression of the recording for the electroencephalographer, as indicated in the CAET minimal technical standards. ${ }^{23}$

\section{EEG REQUISITION, RECORDS, REPORTS}

\section{EEG Requisition}

All EEG requisitions must contain the following: date and time of the recording, patient's name, home address, phone number, date of birth, personal health number, name of referring physician, clinical indication for the test, and medications; and any special requests for additional procedures, sleep deprivation, or additional electrode placement. Such additional information as contamination risk (i.e., methicillin-resistant Staphylococcus aureus, vancomycin-resistant Enterococcus, and suspicion of Creutzfeldt-Jakob disease) should be included so that appropriate laboratory protocols can be adhered to. For inpatient referrals, the unit should be included. ${ }^{6,23}$

\section{The EEG Report}

All EEG reports should meet ACNS minimum reporting standards. The report should comprise three main sections: introduction, description, and interpretation. ${ }^{51}$ Requests for urgent interpretations and critical test results identified by the technologists should be given priority.

It is possible to read an EEG from such portable devices as tablets, cellular phones, and similar devices in order to give a preliminary report; however, a monitor with appropriate screen resolution is recommended for final interpretations. When interpreting topographic mapping, frequency or power spectral analyses and other quantitative assessments of a digital EEG should also involve assessment of the standard EEG. The use of quantitative EEGy alone can yield misleading information. ${ }^{7}$

\section{Introduction}

Formal typed reports must include: department name, address and phone number, date of procedure, date of transcription, patient name, date of birth, hospital number, EEG laboratory identification number, name of referring physician, electroencephalographer's name, whether or not the contents of the report have been verified by the author, the initials of the technologists, and the initials of the transcriptionist.

The report must also include: clinical reasons for the test as per the referring physician, relevant medications and dosages, and state of the patient (e.g., awake, drowsy, asleep, coma, druginduced state). Other channels of physiologic display should be described, such as those reserved for measurement of the ECG, breathing, as well as limb and chin movements. Reasons should be given if the electrode positions or recording techniques vary from the routine laboratory protocol. Documentation should also be provided to indicate where an EEG is being performed, if outside the EEG laboratory, use of any special electrodes or techniques, and conditions prevalent at the time of recording (e.g., fasting, SD, and sedatives or other drugs used for the recording). Reporting the duration of the recording should also be considered if it deviates from a typical recording. ${ }^{51}$

\section{Description of EEG Activity}

The description of the EEG should include the normal and abnormal characteristics of the recording, presented in an objective manner. The purpose for this is to produce a complete report that will allow another electroencephalographer to arrive at a similar conclusion without the benefit of looking at the EEG. ${ }^{51,52}$ The report should describe the background rhythms and reactivity (normal or otherwise), as well as other normal and abnormal nonrhythmic phenomena. Background activity should be described along with its frequency, quantity, amplitude, location, persistence (continuous or intermittent), symmetry, and rhythmicity. ${ }^{51,52}$ Sleep staging should always be described.

The frequency of any EEG phenomenon should be given in hertz, and the estimated mean amplitude should be given in microvolts. ${ }^{51,52}$ Descriptions such as low, medium, and high amplitude are also acceptable. In order to facilitate inter-reader agreement, the report should include either the exact time of key abnormalities or whether such abnormalities are marked on the recording.

In the description of activation procedures, a statement should be included pertaining to their quality (e.g., good, fair, or poor HV). The type and range of frequencies of IPS used should be stated. If HV and IPS are not performed, the reason for this omission should be documented. Normal and abnormal responses to background activation procedures should be described (e.g., touch, sound, eye opening, nasal tickling, mouth or tracheal suctioning, or sternal pressure).

The presence of artefacts should be noted when they interfere with reliable interpretation, when they are questionable and could represent cerebral activity, when they are unusual or excessive, and when they may provide valuable diagnostic information (e.g., myokymia, nystagmus). ${ }^{51,52}$

When a focal abnormality is reported, it should be described by brain region or more precisely by electrode involvement. ${ }^{51,52}$ If an electro-clinical seizure or other clinical event is recorded, a detailed clinical description of the event and any associated EEG changes should be provided.

\section{Clinical Interpretation}

The interpretation should be concise, clear, and understandable by any healthcare practitioner who receives the report. ${ }^{52}$ The report should include a statement of EEG normality or 
abnormality relevant to the clinical problem, correlation of the abnormality with past recordings, diagnosis if apparent, and potential differential diagnoses. If the record is considered abnormal, it is desirable to grade the abnormality in order to facilitate comparison between successive records. When dealing with several types of abnormal features, the list should be limited to the two or three most important abnormalities. In cases where the EEG is strongly suggestive of a certain condition that is not mentioned in the clinical history, it is prudent to mention the fact that such EEG abnormalities are frequently found in association with the clinical condition (i.e., generalized periodic discharges suggesting Creutzfeldt-Jakob disease in a patient with cognitive deterioration but are not necessarily indicative of it). ${ }^{51}$ In general, a normal record does not require further explanation. ${ }^{51}$ Appropriate feedback to the referring physician should be provided. The electroencephalographer should not suggest changes in medications, new treatments, or other clinical tests in the EEG report, with the exception of repeating the EEG with SD and sleep recording when a suspicious finding may result in an unquestionable one during sleep (i.e., focal IEDs). If prior EEGs are available, comparison with those tracings must be discussed. ${ }^{51}$

\section{Classification of EEGs}

To our knowledge, there are no guidelines, recommendations, consensus, or standardized classifications of routine scalp EEG recording at the present time. As such, it is not mandatory to use an EEG classification system. An old and arbitrary classification of the EEG in post-cardiac-arrest patients is sometimes extrapolated to other aetiologies and is still presently used. ${ }^{14,53}$ Other centres prefer the dichotomy of normal versus abnormal result followed by an enumeration of the abnormalities found, while others may use a degree of abnormalities (i.e., mild, moderate, and severe or numeric degree ranging from 0 to 5). A classification system offers the advantage to the neurophysiologist and clinician of quickly identifying the nature/extent of an abnormality, and it facilitates comparisons between recordings in the same patient. A consistent form of classification should be standardized within each laboratory at the discretion of the laboratory director. ${ }^{6}$

\section{Timeline for Reporting EEGs}

Currently, no guidelines for a minimal reporting time exist for EEGy. It is recommended that the timing of a report be based on the purpose and findings of the EEG. An outpatient routine EEG report is expected to be available within five working days of completion. If significant abnormalities exist (e.g., a recorded seizure), the report should be generated within 24 hours of completion with immediate notification to the referring physician. Inpatient routine EEG reports should be generated within 24 hours. In addition, preliminary reports (either verbal or via the hospital electronic/paper healthcare record) should be promptly given to healthcare providers when clinically indicated. Potential indications could include an abnormal or unexpected result or patients in a critical-care unit or emergency department, where timely EEG reports can alter patient management. It is recommended for such long-term EEG recordings as inpatient cEEG/ LTME or AEEG that the typed/signed report be available to the referring physician within 10 days of the EEG. ${ }^{6}$ Reports from such invasive EEG procedures as electrocorticography or intracranial EEG should be available as per local institutional protocols.
Continuous electroencephalograms, LTME, and invasive EEG recordings should be reviewed and reported on a daily basis to the electroencephalographer, EEG technologist, attending physician, and nurses. The first hour of cEEG recording should be reviewed immediately, and a verbal report provided after it is acquired. ${ }^{14,39-41}$ Ideally, remote access to the cEEG by the electroencephalographer should be a requirement. Unfortunately, this is not available at all centres across Canada.

\section{STORAGE OF RECORDS}

Retention, storage, and disposal of the legal typed reports and actual recordings must be maintained as per the local facility, health region, or provincial policies and the mandatory regulations while ensuring patient confidentiality. ${ }^{22}$ Laboratories should retain a master list of EEG tests that includes all the names of patients tested, their identifying numbers, and dates of the test and the EEG report. ${ }^{6}$ All records should meet the guidelines for medical records as stated in the Public Hospital's Act for each province. ${ }^{7}$ Considering that epilepsy may continue for decades, it is reasonable for electroencephalography laboratories to keep pruned abnormal recordings for up to 25 years or longer.

For inpatient long-term recordings lasting 24 hours or more, storage on a digital server system is recommended, which can facilitate review in remote locations and backup by the hospital's information technology staff. Storage of video EEG data via business-grade server storage solutions minimizes the chance of data loss by incorporating built-in data storage redundancy and regular data backup. Additionally, using a server storage solution enables full Health Insurance Portability and Accountability Act compliance because it can record a full audit trail for every person who accesses the patient record. Optical storage devices are not recommended. ${ }^{47}$

\section{SAFETY}

Seizures are potentially dangerous events that can lead to serious injury or death. It is therefore recommended by the ILAE to have safety protocols or manuals in place. ${ }^{54}$ It is expected that all personnel adhere to Health Canada Guidelines and the Canadian Occupational Health and Safety Act. Interdisciplinary team members must comply with their governing body's code of ethics.

Collodion is one of the best techniques for securing EEG electrodes to the scalp, yielding superior recording quality for prolonged studies such as LTME and the ICU. Acetone is the most effective agent for removing collodion-applied electrodes; however, acetone inhalation can produce nasal and conjunctival irritation, respiratory effects, nausea and vomiting, and a sensation of muscle weakness. Proper ventilation is required when using ether-based products (e.g., collodion) as per the Material Safety Data Sheet. It has been demonstrated that a simple vapour extraction system available during electrode application and removal improves safety in EEG laboratories. ${ }^{55}$ On the other hand, the use of ether-based products such as collodion is not recommended for electrode application in the routine 30-minute studies. $^{22}$

\section{EEG Laboratory Manuals}

An EEG manual provides a platform with which to assist in achieving and maintaining study quality and helps a laboratory 
meet the current standard of care. Every staff member is responsible for providing and maintaining safe patient care, infection prevention and control, safe transferring/lifting and moving, and equipment, as well as electrical and fire safety. In addition to occupational safety and emergency preparedness, personnel must comply with their local facilities' occupational health and safety policies and procedures.

Current practices indicate that the medical director or designate has the ultimate responsibility for the policies and procedures laboratory manual. All procedures should be initially approved and signed by the medical director. The document should be reviewed annually by the director or a designate. The review should include the documentation of any changes made during the year. Table 1 summarizes the information that should be contained in the manual. ${ }^{7,24}$

\section{Infection Control}

The principles of Standard Precautions and Transmission Based Procedures (formerly "universal precautions") should be respected. ${ }^{6}$ The technologists who employ needle electrodes need training on the use, disposal, and advantages/disadvantages of their use. ${ }^{32}$ Needle electrodes must not be used in routine clinical settings and never used in out-of-hospital laboratories. If exceptional circumstances necessitate their use (burns to the head, intraoperative procedures), sterilized single-use needle electrodes should be used and disposed of after testing has been completed, and CAET guidelines should be enforced. ${ }^{7,22}$ Electrodes in patients with Creutzfeldt-Jakob disease should be disposed of. Reducing skin impedance should be done in accordance with the current CAET minimal technical standards. ${ }^{22}$

Soiled medical devices should be handled in a manner that reduces the risk of exposure and/or injury to personnel and clients/ patients/residents, or of contamination of environmental surfaces. Cleaning is always required prior to disinfection and/or sterilization. An item that has not been cleaned cannot be adequately disinfected or sterilized. Disk electrodes as applied for routine EEG procedures are classified as semi-critical (due to skin abrasion), requiring high-level disinfection as per the facility's infection control policies. 56,57

It is highly recommended that electrode caps with predetermined electrode positions not be used. If no other options exist (e.g., in exceptional circumstances when patient cooperation is such that use of an electrode cap is the only way to obtain an EEG), then such a system may be used. When using an electrode cap, the technologist must clearly document this on the recording. ${ }^{22}$

\section{Electrical Safety in Special Care Units:}

When indwelling catheters or pacemaker electrodes are connected to the patient, special precautions must be taken to ensure that all these connections are properly isolated or current-limited. It is strongly recommended that electrical interference be promptly investigated, as this may be indicative of current leakage through the patient. ${ }^{44}$

\section{Informed Consent}

All patients, or their appointed decision makers, should make an informed decision before having an EEG performed. Written

\section{Table 1: EEG department manuals}

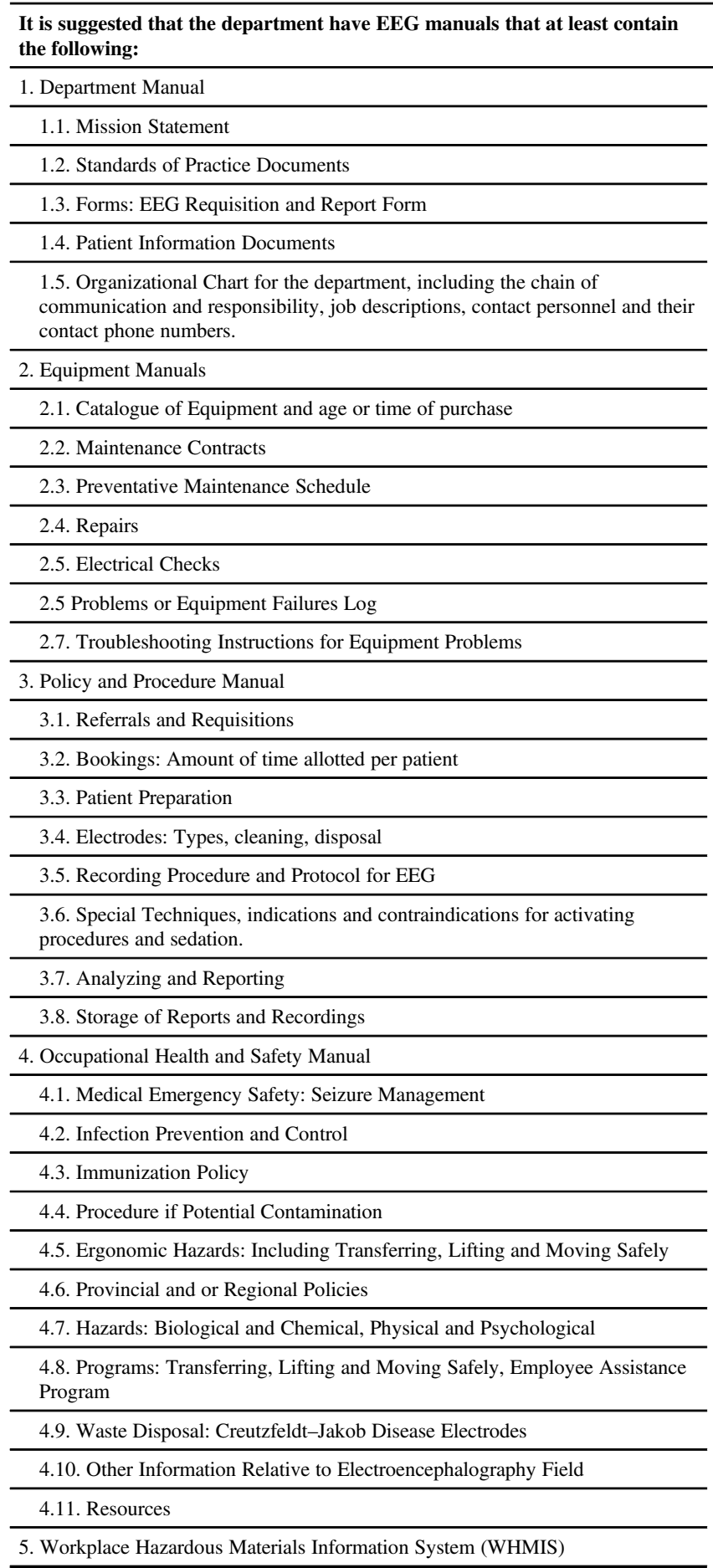

consent is not necessary for routine EEGs. The patient/appointed decision maker should receive adequate information about the purpose of the EEG (being as specific as is necessary), the nature of the test, the procedures used, and the potential risks and benefits. All patients or their substitute decision makers should be informed that they have the right to consent to or refuse the test in whole or in part at any time during the procedure without 
prejudice. When activating procedures (i.e., reduction of medications, SD, or use of sedative drugs) put the patient at risk of seizures, excessive sedation, or other complications, it is recommended that the patients or their caregivers be at least verbally informed about this. It is strongly recommended that a factsheet that provides the aforementioned general information be utilized for elective EEGs. In special situations such as LTME/cEEG or invasive procedures (i.e., insertion of depth or subdural electrodes), written consent is strongly recommended. ${ }^{6}$

\section{Quality Assurance}

The purpose of a quality-assurance program is to assure the quality of practice of the electroneurophysiology laboratory and to promote continuing evaluation, competence, and improvements. The process should monitor participation and compliance with established professional and departmental practices and should focus on patient quality and safety as well as the services provided. Quality-assurance checks can be completed quarterly or semiannually to identify areas of concern. Appropriate documentation and a collaborative, continuous improvement process approach is beneficial. ${ }^{24,32}$ The program should be efficient, standardized, and easy to implement, and it should develop/ employ procedures that allow easy acquisition of qualityassessment data. ${ }^{58}$ All the responsibility and integrity of a quality-assurance program and nearly all the effort and expense should lie within each laboratory.

\section{Quality Assurance Parameters}

The program should continually monitor, at minimum, the parameters listed below.

\section{Patient Satisfaction}

An anonymous and unbiased mail return or electronic evaluation provides valuable feedback that may guide improvements that need to be implemented. This should be undertaken to more fully reflect the needs and satisfaction of patients, staff, and referring medical officers. This is actively encouraged at all levels to ensure that program objectives and client needs are met consistent with governing policies. ${ }^{59}$

\section{Procedures and Processes}

An audit should be part of all clinical services. Audits examine and reflect the range of procedures undertaken, their quality, and outcome, and they provide evidence of their benefits. ${ }^{60}$ Evaluations for all procedures and processes, as set forth by the most current standards of practice in accordance with the CAET, CBRET, and CSCN, and by the local facility, should continue to be part of the continual evaluation and improvement process.

\section{Staff Competence}

All professionals have a registration process with competencies that may be used as guidelines for maintaining qualityassurance competency. Research should be encouraged because it improves knowledge and standards of care. ${ }^{60}$

\section{Staff Compliance}

Each facility should implement an ongoing evaluation process by which staff compliance to their field of practice standards and guidelines are assessed and documented for continuous quality- assurance improvements. Increased awareness of departmental quality assurance, program evaluations, and staff participation should increase the quality of services.

\section{Equipment}

All equipment and testing should be performed in accordance with CAET standards and CSA standards, and used in accordance with the manufacturer's recommendations.

\section{Analyzing and Reporting: Technologist, Electroencephalographer, Nurses, Medical Office Assistants}

Each facility should establish an accurate quality-assurance process that covers data acquisition, data interpretation, reporting, transcription, and dissemination of verbal or written reports as per their variations in clinical practice. The facility standard should reflect the agreed-upon standard turnaround time between reception of the EEG requisition to delivery of the results. Every step in the process should be evaluated for continuous improvements. Delays in critical test results may result in serious adverse outcomes for patients. ${ }^{32,61}$

\section{Safety}

All facilities should evaluate their department's adherence to the national and local facility safety standards, including local facility infection control policies

\section{Patient Access and Waitlists}

For the provision of services, each lab must have access (direct or indirect via referral) to the following ${ }^{60}$ :

- Local dedicated epilepsy service

- Specialized interdisciplinary team

- Inpatient facilities for emergencies (i.e., status epilepticus)

- Laboratory tests and therapeutic drug monitoring

- Range of imaging techniques, particularly MRI

- Epilepsy surgery program

- Special assessment unit for intermediate care

- Assessment by psychologists specializing in epilepsy

- Epilepsy helpline and readily available contact with voluntary organizations

- Readily available and easily understood written information about seizures, types of epilepsy, investigations and treatment

The length of time a patient remains on a waitlist should be monitored for continuous improvement. Acceptable waitlist timeframes should be established by departments, local facilities, health regions, or within the province.

\section{ConClusions}

Fifteen years have passed since the Task Force of the Canadian Society of Clinical Neurophysiologists published in 2002 the Canadian electroencephalography standards, an initial set of guidelines for electroencephalography in Canada. We present herein an updated version with the minimal standards for the practice of conventional EEG. The purpose of these minimal standards will serve as a benchmark for further development, standardization, and quality care in clinical neurophysiology laboratories in Canada. 


\section{AbBreviations}

ACNS = American Clinical Neurophysiology Society; $\mathrm{AEEG}=$ ambulatory $\mathrm{EEG} ; \mathrm{CAET}=$ Canadian Association of Electroneurophysiology Technologists; $\quad$ CBRET $=$ Canadian Board of Registration of Electroencephalographic Technologists Inc.; cEEG = continuous electroencephalogram; CSA = Canadian Standards Association; $\mathrm{CSCN}=$ Canadian Society of Clinical Neurophysiologists; $\mathrm{ECG}=$ electrocardiogram; $\mathrm{EEG}=$ electroencephalogram; EEGy = electroencephalography; EMG = electromyogram; GPDs = generalized periodic discharges; $\mathrm{HV}=$ hyperventilation; ICU = intensive care unit; IEDs = interictal epileptiform discharges; ILAE = International League Against Epilepsy; IPS = intermittent photic stimulation; LTME = long-term monitoring for epilepsy; NICE $=$ National Institute for Health and Clinical Excellence; PNES=psychogenic non-epileptic seizure; $\mathrm{SD}=$ sleep deprivation.

\section{Disclosures}

All of the authors hereby declare that they have nothing to disclose.

\section{SUPPLEMENTARY MATERIALS}

To view the supplementary materials for this article, please visit https://doi.org/10.1017/ cjn.2017.217.

\section{REFERENCES}

1. Velis D, Plouin P, Gotman J, et al. Recommendations regarding the requirements and applications for long-term recordings in epilepsy. Epilepsia. 2007;48(2):379-84.

2. Andre-Obadia N, Parain D, Szurhaj W. Continuous EEG monitoring in adults in the intensive care unit (ICU). Neurophysiol Clin. 2015;45(2):39-46.

3. Varelas PN, Spanaki MV, Hacein-Bey L, Hether T, Terranova B. Emergent EEG: indications and diagnostic yield. Neurology. 2003;61(5):702-4.

4. Velis D, Plouin $P$, Gotman $J$, da Silva FL, ILAE DMC. Subcommittee on Neurophysiology. Recommendations regarding the requirements and applications for long-term recordings in epilepsy. Epilepsia. 2007;48(2):379-84.

5. Andrade DM, Tai P, Dalmau J, Wennberg R. Tonic seizures: a diagnostic clue of anti-LGI1 encephalitis? Neurology. 2011; 76(15):1355-7.

6. Smith SJ. EEG in the diagnosis, classification, and management of patients with epilepsy. J Neurol Neurosurg Psychiatry. 2005; 76(Suppl 2):ii2-7.

7. Task Force of the Canadian Society of Clinical Neurophysiologists. Minimal standards for electroencephalography in Canada. Can J Neurol Sci. 2002;29(3):216-20.

8. Lagerlund TD, Worrell JA. The role of routine scalp electroencephalography. In: Cascino GD, Sirven JL editors Adult Epilepsy. New York: John Wiley \& Sons; 2011, P. 27-48.

9. Maganti RK, Rutecki P. EEG and epilepsy monitoring. Continuum (Minneap Minn). 2013;19(3 Epilepsy):598-622.

10. Bihege CJ, Langer T, Jenke AC, Bast T, Borusiak P. Prevalence of epileptiform discharges in healthy infants. J Child Neurol. 2015;30(11):1409-13.

11. Borusiak P, Zilbauer M, Jenke AC. Prevalence of epileptiform discharges in healthy children: new data from a prospective study using digital EEG. Epilepsia. 2010;51(7):1185-8.

12. Reeves RR, Ladner ME. Effect of inpatient electroencephalography on clinical decision making: EEG is more valuable than findings suggest. J Am Osteopath Assoc. 2014;114(6):425-6.

13. Rowan AJ. The utility of EEG. In: Rowan AJ, Tolunsky E editors. Primer of EEG: With a Mini-Atlas. Philadelphia: Elsevier Science; 2003.
14. Herman ST, Abend NS, Bleck TP, et al. Consensus statement on continuous EEG in critically ill adults and children, part 1: indications. J Clin Neurophysiol. 2015;32(2):87-95.

15. Westhall E, Rossetti AO, van Rootselaar AF, et al. Standardized EEG interpretation accurately predicts prognosis after cardiac arrest. Neurology. 2016;86(13):1482-90.

16. Stecker MM, Sabau D, Sullivan L, et al. American Clinical Neurophysiology Society guideline 6: minimum technical standards for EEG recording in suspected cerebral death. J Clin Neurophysiol. 2016;33(4):324-7.

17. Coburn KL, Lauterbach EC, Boutros NN, Black KJ, Arciniegas DB, Coffey CE. The value of quantitative electroencephalography in clinical psychiatry: a report by the Committee on Research of the American Neuropsychiatric Association. J Neuropsychiatry Clin Neurosci. 2006;18(4):460-500.

18. Sutter R, Kaplan PW, Cervenka MC, et al. Electroencephalography for diagnosis and prognosis of acute encephalitis. Clin Neurophysiol. 2015;126(8):1524-31.

19. Guerrini R, Duchowny M, Jayakar P, et al. Diagnostic methods and treatment options for focal cortical dysplasia. Epilepsia. 2015;56 (11):1669-86

20. Schmitt SE, Pargeon K, Frechette ES, Hirsch LJ, Dalmau J, Friedman D. Extreme delta brush: a unique EEG pattern in adults with anti-NMDA receptor encephalitis. Neurology. 2012;79(11): 1094-100.

21. Badrakalimuthu VR, Swamiraju R, de Waal H. EEG in psychiatric practice: to do or not to do? Adv Psychiatr Treat. 2016;17(2): $114-21$

22. Canadian Association of Electroneurophysiology Technologists. CAET minimal technical standards: routine adult, routine paediatric, routine neonatal, routine ECS. Saskatoon, Saskatchewan: CAET; 2016. http://www.caet.org/joomlacaet/. Accessed June 7, 2017.

23. Canadian Board of Registration Electroencephalograph Technologists; 2016. www.CBRET.org. Accessed June 7, 2017.

24. Alberta College of Medical and Diagnostic and Therapeutic Technologists. Core competencies for electroencephalography; 2016. www.acmdtt.com. Accessed June 7, 2017.

25. American Clinical Neurophysiology Society. Guideline twelve: guidelines for long-term monitoring for epilepsy. J Clin Neurophysiol. 2008;25(3):170-80.

26. American Society of Electroneurodiagnostic Technologists Inc. National competency skill standards for ICU/cEEG monitoring. Am J Electroneurodiagnostic Technol. 2008;48(4):258-64.

27. Buelow JM. Kathleen Mears Memorial Lecture: an update on patient safety issues in the epilepsy monitoring unit. Neurodiagn J. 2013;53(2):104-13.

28. Wirrell E, Kozlik S, Téllez J, Wiebe S, Hamiwka L. Ambulatory electroencephalography (EEG) in children: diagnostic yield and tolerability. J Child Neurol. 2008;23(6):655-62.

29. Dash D, Hernandez-Ronquillo L, Moien-Afshari F, Téllez-Zenteno JF. Ambulatory EEG: a cost-effective alternative to inpatient video-EEG in adult patients. Epileptic Disord. 2012;14(3): 290-7.

30. Flink R, Pedersen B, Guekht AB, et al. Guidelines for the use of EEG methodology in the diagnosis of epilepsy. International League Against Epilepsy: commission report. Commission on European Affairs: Subcommission on European Guidelines. Acta Neurol Scand. 2002;106(1):1-7.

31. Craciun L, Varga ET, Mindruta I, et al. Diagnostic yield of five minutes compared to three minutes hyperventilation during electroencephalography. Seizure. 2015;30:90-2.

32. American Electroencephalographic Society. Guideline one: minimum technical requirements for performing clinical electroencephalography. J Clin Neurophysiol. 1994;11(1):2-5.

33. American Electroencephalographic Society. Guideline four: standards of practice in clinical electroencephalography. J Clin Neurophysiol. 1994;11(1):14-5.

34. Canadian Society of Clinical Neurophysiologists. Guidelines for visual-sensitive EEG testing. Can J Neurol Sci. 2008;35(2):133-9.

35. Seshia SS, Carmant L. Visual-sensitive epilepsies: classification and review. Can J Neurol Sci. 2005;32(3):298-305. 
36. Giorgi FS, Maestri M, Guida M, et al. Controversial issues on EEG after sleep deprivation for the diagnosis of epilepsy. Epilepsy Res Treat. 2013;2013:614-85.

37. Giorgi FS, Perini D, Maestri M, et al. Usefulness of a simple sleepdeprived EEG protocol for epilepsy diagnosis in de novo subjects. Clin Neurophysiol. 2013;124(11):2101-7.

38. Sinha SR, Sullivan L, Sabau D, et al. American Clinical Neurophysiology Society guideline 1: minimum technical requirements for performing clinical electroencephalography. J Clin Neurophysiol. 2016;33(1):303-7.

39. Jette N, Claassen J, Emerson RG, Hirsch LJ. Frequency and predictors of nonconvulsive seizures during continuous electroencephalographic monitoring in critically ill children. Arch Neurol. 2006;63(12):1750-5.

40. Schreiber JM, Zelleke T, Gaillard WD, Kaulas H, Dean N, Carpenter JL. Continuous video EEG for patients with acute encephalopathy in a pediatric intensive care unit. Neurocrit Care. 2012;17(1):31-8.

41. Claassen J, Mayer SA, Kowalski RG, Emerson RG, Hirsch LJ. Detection of electrographic seizures with continuous EEG monitoring in critically ill patients. Neurology. 2004;62(10):1743-8.

42. André M, Lamblin MD, d'Allest AM, et al. Electroencephalography in premature and full-term infants: developmental features and glossary. Neurophysiol Clin. 2010;40(2):59-124.

43. Okumura A. The diagnosis and treatment of neonatal seizures. Chang Gung Med J. 2012;35(5):365-72.

44. Tyner FS. Fundamentals of EEG Technology: Clinical Correlates. Philadelphia: Raven Press; 1989.

45. Acharya JN, Hani A, Cheek J, et al. American Clinical Neurophysiology Society guideline 2: guidelines for standard electrode position nomenclature. J Clin Neurophysiol. 2016;33(4):308-11.

46. Acharya JN, Hani AJ, Thirumala PD, et al. American Clinical Neurophysiology Society guideline 3: a proposal for standard montages to be used in clinical EEG. J Clin Neurophysiol. 2016;33(4):312-6.

47. Halford JJ, Sabau D, Drislane FW, Tsuchida TN, Sinha SR. American Clinical Neurophysiology Society guideline 4: recording clinical EEG on digital media. J Clin Neurophysiol. 2016;33:317-9.

48. American Clinical Neurophysiology Society. Guideline 8: guidelines for recording clinical EEG on digital media. J Clin Neurophysiol. 2006;23(2):122-4.
49. American Clinical Neurophysiology Society. Guideline 6: a proposal for standard montages to be used in clinical EEG. J Clin Neurophysiol. 2006;23(2):111-7.

50. Blume WT. The necessity for sphenoidal electrodes in the presurgical evaluation of temporal lobe epilepsy: con position. J Clin Neurophysiol. 2003;20(5):305-10.

51. American Clinical Neurophysiology Society. Guideline 7: guidelines for writing EEG reports. J Clin Neurophysiol. 2006;23(2): 118-21.

52. Kaplan PW, Benbadis SR. How to write an EEG report: dos and don'ts. Neurology. 2013;80(Suppl 1):S43-6.

53. Hockaday JM, Potts F, Epstein E, Bonazzi A, Schwab RS. Electroencephalographic changes in acute cerebral anoxia from cardiac or respiratory arrest. Electroencephalogr Clin Neurophysiol. 1965;18:575-86.

54. Labiner DM, Bagic AI, Herman ST, et al. Essential services, personnel, and facilities in specialized epilepsy centers: revised 2010 guidelines. Epilepsia. 2010;51(11):2322-33.

55. Young B, Blais R, Campbell V, et al. Vapors from collodion and acetone in an EEG laboratory. J Clin Neurophysiol. 1993;10 (1):108-10.

56. American Society of Electroneurodiagnostic Technologists. National competency skill standards for long-term monitoring in epilepsy. Am J Electroneurodiagnostic Technol. 2005;45(1):61-71.

57. Scott CA, Fish TR, Allen PJ. Design of an intensive epilepsy monitoring unit. Epilepsia. 2000;41(Suppl 5):S3-8.

58. Stocker T, Schneider F, Klein M, et al. Automated quality assurance routines for fMRI data applied to a multicenter study. Hum Brain Mapp. 2005;25(2):237-46.

59. Hamilton-Bruce MA, Black AB, Stratos K. Quality assurance in a neurophysiology laboratory. Australas Phys Eng Sci Med. 1994;17(2):94-5.

60. Brodie MJ, Shorvon SD, Canger R, et al. Commission on European Affairs: appropriate standards of epilepsy care across Europe-ILEA. Epilepsia. 1997;38(11):1245-50.

61. Massachusetts Coalition for Prevention of Medical Errors. 2017, Patient Safety Forum. Cambridge: Massachusetts Coalition for Prevention of Medical Errors; 2017. http://www.macoalition. org/patient-safety-forum-3-2017.shtml. Accessed June 7, 2017. 